\title{
Quality of Life following Total Laryngectomy: Assessment Using the UW-QOL Scale
}

\author{
R. Kazi ${ }^{a, b} \quad J . D^{2}$ Cordova ${ }^{a} \quad J . K^{2}$ anagalingam ${ }^{a} \quad$ R. Venkitaraman ${ }^{a} \quad$ C.M. Nutting ${ }^{a}$ \\ P. Clarke ${ }^{a}$ P. Rhys-Evans ${ }^{a} \quad$ K.J. Harrington ${ }^{a, b}$ \\ ${ }^{\mathrm{a} H e a d}$ and Neck Unit, Royal Marsden Hospital, and ${ }^{\mathrm{b}}$ The Institute of Cancer Research, London, UK
}

\section{Key Words}

Quality of life - Total laryngectomy · University of

Washington Quality of Life Scale

\begin{abstract}
Objectives: To determine the quality of life $(\mathrm{QOL})$ in patients using valved speech following total laryngectomy with a validated patient self-report scale. Study Design: Cross-sectional cohort study. Patients: 63 patients following total laryngectomy using valved speech. Intervention: University of Washington Quality of Life (UW-QOL) questionnaire. Main Outcome Measures: Patient perception of the QOL over the last 7 days following total laryngectomy in response to specific questions and correlated with sociodemographic and treatment factors. Results: Responses were received from 44 males and 11 females (response rate: $87.3 \%$ ) with a median age of 66 years (range: $40-84$ ). The mean (SD) composite score of the QOL in our series of patients with total laryngectomy was 81.3 (10.9). As regards the overall QOL, $80 \%$ of our patients cited it as very good $(45.5 \%)$ to good $(34.5 \%)$. Patients identified speech, appearance, and activity as the most important issues following total laryngectomy. Only age and pharyngo-oesophageal segment closure were significant predictors of QOL scores (Student $t$ test, $\mathrm{p}<0.05$ ) and not other demographic and treatment variables. Conclusions: The composite score and overall QOL were high in
\end{abstract}

our series of total laryngectomy patients and this possibly reflects adequate multidisciplinary management. Westrongly urge the use of prospective longitudinal studies that will adequately identify any QOL changes over time. Although the UW-QOL questionnaire is a simple and brief scale, it has limitations that can curtail its effective use in laryngectomy patients and we advise supplementing it with the use of domain-specific questionnaires. Copyright $\odot 2007$ S. Karger AG, Basel

\section{Introduction}

Quality of life (QOL) is a multidimensional construct that minimally includes broadly defined assessments of the physical, psychological, and social domains of functioning [1]. Patients with head and neck cancer (HNC) are rendered vulnerable to psychosocial problems because social interactions and emotional expression depend to a great extent upon the structural and functional integrity of the head and neck region. The impact of a diagnosis of $\mathrm{HNC}$ and the consequences of treatment cross multiple functional domains that have a clear and direct influence on well-being and associated QOL. Length of survival alone is an unsatisfactory measure of the success of treatment; the quality of survival needs to be evaluated. The evaluation of QOL and performance outcome in

\section{KARGER}

Fax +41613061234 E-Mail karger@karger.ch www.karger.com
(C) 2007 S. Karger AG, Basel

0301-1569/07/0692-0100\$23.50/0

Accessible online at:

www.karger.com/orl
Dr. Rehan Kazi

Head and Neck Unit

Royal Marsden Hospital, 203 Fulham Road

London SW3 6JJ (UK)

Tel. +44 207808 2732, Fax +44 207808 2235, E-Mail rehan.kazi@rmh.nhs.uk 
HNC is critical to optimal patient care, comprehensive evaluation of treatment alternatives, and the development of informed rehabilitative services and patient education. However, measuring QOL is complicated by the fact that there are many different validated questionnaires available [2]. In addition, QOL is an individual perception that can be affected by health status, as well as by disease. When quantifying QOL, we must consider the influence of numerous factors and their potential interdependence if accurate representations of QOL status are to be gathered [3].

In the case of laryngeal cancer, results from the use of QOL scales highlight the fact that although treatment of the cancer is successful, individuals continue to experience difficulties in daily activities and social participation, regardless of the type of treatment (i.e. radiation therapy, conservative or radical surgery, chemotherapy, or a combined treatment protocol) [4]. This is particularly true when treatment requires total laryngectomy (TL). TL will result in significant levels of change in the physical, psychological, social, and emotional domains with an ultimate influence on the individual's judgment of his or her own QOL [4]. Functional restrictions in these domains are further complicated by the fact that TL results in complete loss of normal verbal communication. Although objective values of tracheo-oesophageal (TO) speech often fall within the normal laryngeal range, listeners clearly identify TO speakers as being perceptually less acceptable and less intelligible than normal laryngeal speakers or those who have been treated with radiotherapy $[3,5]$. There is a perception that TL has a devastating effect on patients and their family members because of the presence of a definitive stoma and the loss of the larynx. These results have direct implications on social acceptance and interaction, functional communication, and the adjustment of individuals who undergo TL. Consequently, the psychosocial impact of such concerns cannot be disregarded.

The impact on QOL after TL may change, depending on the time of analysis, and may be influenced by factors such as fear of recurrence, difficulties in voice production, and side effects of chemotherapy and radiotherapy. However, when QOL has been assessed with multidomain questionnaires, the results surprisingly seem to indicate that functional limitations caused by a laryngectomy do not necessarily result in a lower overall QOL [3-5]. Although there is evidence that with time patients adjust and learn to cope with the disease and treatment sequelae, few studies have addressed the long-term QOL after TL $[3,5-8]$.

Quality of Life following Total Laryngectomy
The impact of a TL on QOL needs to be examined more carefully using patient self-report scales. Much of the work done before has been handicapped either by the small patient numbers, variety of assessment scales used or inadequate data $[3,5,9,10]$. Consequently, the purpose of this investigation was threefold: (1) to determine how effectively QOL can be assessed in individuals who use TO speech as their primary mode of communication using the well-validated University of Washington Head and Neck Quality of Life (UW-QOL) version 4 instrument; (2) to determine sociodemographic factors that influence QOL, and (3) to compare these outcomes with those reported previously in the literature [11-14]. To the best of our knowledge, our assessment using this validated scale is the largest series of its kind in Europe.

\section{Materials and Methods}

We identified 63 patients who had undergone TL from the Royal Marsden Hospital Speech and Language Therapy database. All patients were disease-free and were using a Blom-Singer voice prosthesis during the period of this cross-sectional study. All 63 patients were sent the UW-QOL questionnaire by mail with a personalized covering letter providing information about the survey along with a prepaid return envelope.

The UW-QOL questionnaire is a HNC-specific, self-administered scale that has shown promising reliability, responsiveness, and validity for the assessment of QOL in patients with surgically treated HNC [11-13]. It consists of single questions that address 12 domains over the period of the last 7 days: pain, appearance, activity, recreation, swallowing, chewing, speech, shoulder function, taste, saliva, mood, and anxiety. It also includes a question asking the patient to list the 3 domains that have been most important in the last 7 days and 3 general questions concerning health-related and overall QOL. The domains are scored on a scale ranging from 0 (worst) to 100 (best), to give a composite score. The composite UW-QOL score for this study was computed as the arithmetic mean of the 12 individual domain scores (maximum score, 1,200) $[11,14]$. The global QOL score is a direct overall assessment of QOL and is determined by asking all patients to 'consider everything that contributes to your personal well-being: how would you rate your overall quality of life during the past seven days?' The possible responses here are excellent, very good, good, fair, poor, or very poor.

Time from inception to completion of this study was 3 months. The study was approved by the local research and ethics committee.

\section{Statistical Analysis}

The data were then entered into a worksheet (Excel 05; Microsoft Corp., Washington, D.C., USA) and the means and standard deviations were computed. Statistical analysis was performed using the Statistical Package for Social Sciences 10.0 (SPSS Inc., Chicago version, III., USA). Information from both scales was correlated using Student's t test with sociodemographic and treat- 
Table 1. Treatment and UW-QOL composite score details of the study patients

\begin{tabular}{|c|c|c|c|c|}
\hline \multirow[t]{2}{*}{ Treatment variables } & \multicolumn{2}{|c|}{ Patients } & \multicolumn{2}{|c|}{$\begin{array}{l}\text { UW-QOL com- } \\
\text { posite score }\end{array}$} \\
\hline & $\mathrm{n}$ & $\%$ & mean & SD \\
\hline Primary cases ${ }^{1}$ & 24 & 48.9 & 82.5 & 7.9 \\
\hline Recurrent cases ${ }^{1}$ & 25 & 51 & 79.7 & 10.8 \\
\hline \multicolumn{5}{|l|}{ Site of tumour ${ }^{2}$} \\
\hline Larynx & 37 & 78.7 & 81.4 & 9.22 \\
\hline Hypopharynx & 7 & 14.8 & 83 & 10 \\
\hline Oropharynx & 3 & 6.4 & 77.4 & 9 \\
\hline \multicolumn{5}{|c|}{ Tracheo-oesophageal puncture ${ }^{3}$} \\
\hline Primary & 39 & 75 & 82 & 10 \\
\hline Secondary & 13 & 25 & 81 & 11 \\
\hline \multicolumn{5}{|l|}{ PE segment closure ${ }^{2}$} \\
\hline Circumferential & 11 & 23.4 & 74.5 & 9.6 \\
\hline Horizontal & 31 & 65.9 & 81.9 & 9.6 \\
\hline T-shaped & 4 & 8.5 & 89.2 & 6.8 \\
\hline Myotomy $^{1}$ & 37 & 75.5 & 82 & 8.7 \\
\hline Nerve implantation ${ }^{1}$ & 32 & 65.3 & 81 & 10 \\
\hline Pharyngectomy ${ }^{1}$ & 13 & 26.5 & 82.2 & 9.7 \\
\hline Neck dissection & 24 & 43.6 & 81 & 11.4 \\
\hline Total glossectomy & 3 & 54.5 & 74.3 & 13.2 \\
\hline Reconstruction & 12 & 21.8 & 78.5 & 9.4 \\
\hline Complications & 7 & 12.7 & 76.4 & 11.7 \\
\hline Radiotherapy & 40 & 72.7 & 80.5 & 10 \\
\hline Chemoradiotherapy & 6 & 10.9 & 77.6 & 10 \\
\hline
\end{tabular}

${ }^{1}$ No information in 6 cases.

${ }^{2}$ No information in 8 cases.

${ }^{3}$ No information in 3 cases.

Table 2. Mean and SD scores for each domain

\begin{tabular}{lll}
\hline Domain & Mean & SD \\
\hline Pain & 91.4 & 16.8 \\
Appearance & 78.2 & 19.9 \\
Activity & 76.8 & 18.5 \\
Recreation & 77.3 & 16.9 \\
Swallowing & 84.9 & 17.8 \\
Chewing & 90 & 22.4 \\
Speech & 71.7 & 20.7 \\
Taste & 74.3 & 26.9 \\
Shoulder & 87.3 & 24.4 \\
Saliva & 89.6 & 20.2 \\
Mood & 75.9 & 19.8 \\
Anxiety & 83.3 & 21 \\
\hline Composite score & 81.3 & 10.9 \\
\hline
\end{tabular}

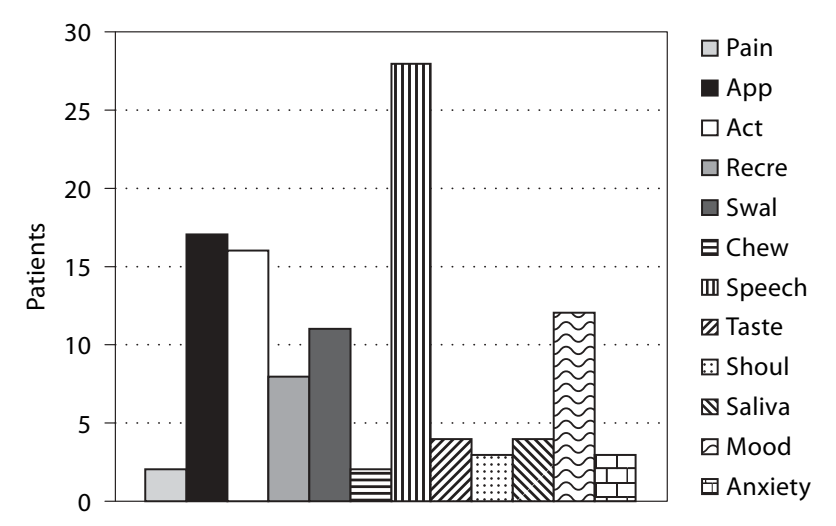

Fig. 1. Issues that were reported as 'most important in the last 7 days' by the study population. App = Appearance; Act = activity; Recre = recreation; Swal = swallowing; Chew = chewing; Shoul $=$ shoulder .

ment variables such as age, sex, neck dissection, primary/recurrent cases, primary/secondary tracheo-oesophageal puncture (TOP), myotomy, nerve implantation, reconstruction procedure, pharyngo-oesophageal (PE) segment closure, chemotherapy, radiotherapy and complications. A p value less than/equal to 0.05 was considered as significant. Results were verified using ANOVA with Bonferroni correction.

\section{Results}

We received 55 completed questionnaires from our initial cohort of 63 patients with a resultant response rate of $87.3 \%$. Most of the questionnaires were obtained in the first week and all by the end of the fourth week. Responses were received from 44 males and 11 females with a median age of 66 years (range: $40-84$ ). All patients were using the Blom-Singer valve. Median time since TL was 81 months (range: 3-268). The treatment details of the study patients are shown in table 1.

The mean (SD) composite QOL score in our series of patients with TL was 81.3 (10.9). We found that our patients scored very well in general and across all the domains, especially in the pain, swallowing, shoulder function, saliva and anxiety domains (see table 2 and fig. 1). Not surprisingly, the speech domain scored the lowest.

Each domain was identified by at least 1 patient as one of the most important issues over the last 7 days. However, in particular, speech ( $\mathrm{n}=28,50.9 \%)$, appearance $(\mathrm{n}=17,30.9 \%)$ and activity $(\mathrm{n}=16,29 \%)$ were most commonly cited (fig. 2). 


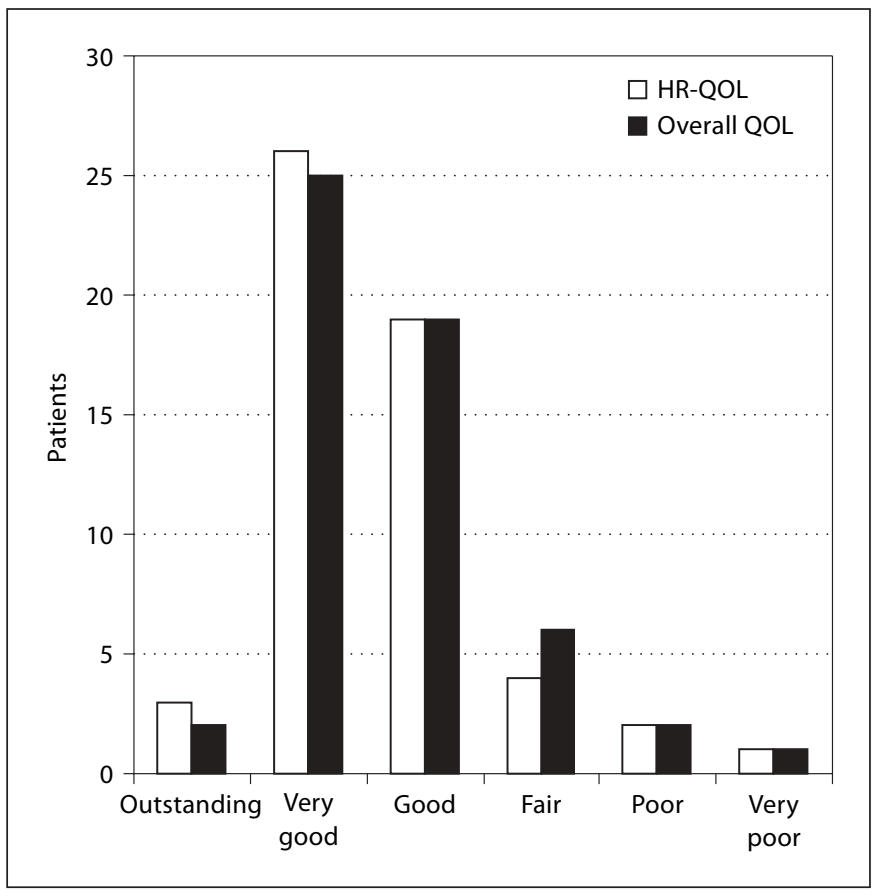

Fig. 2. Health-related QOL (HR-QOL) and global QOL during the past 7 days.

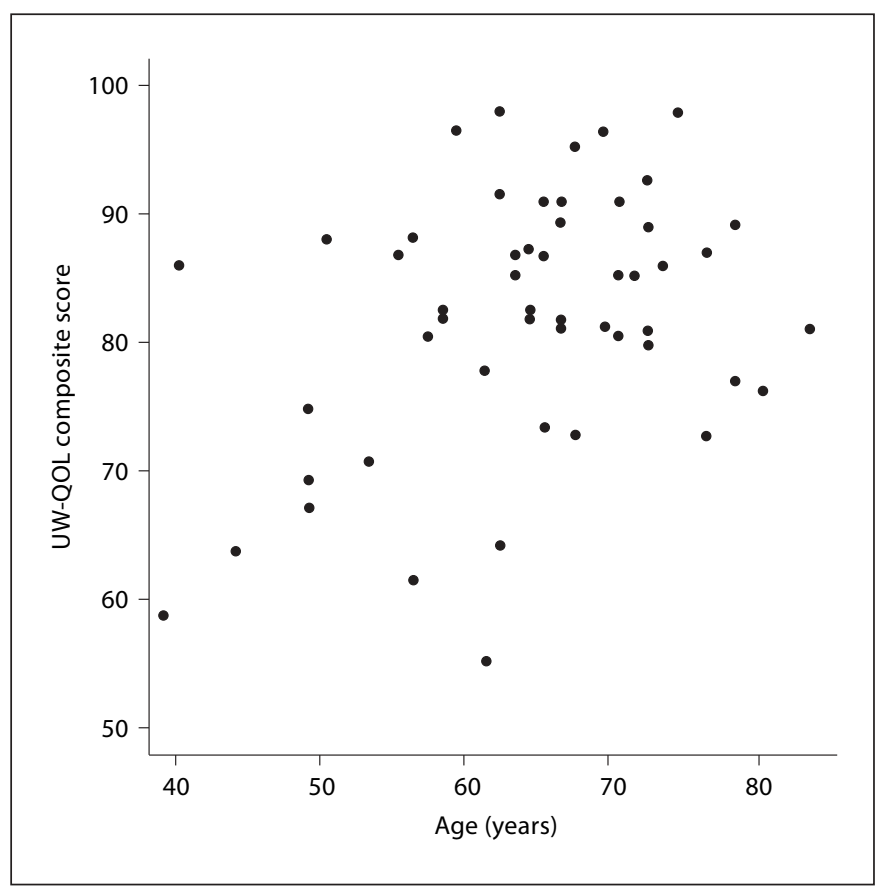

Fig. 3. Mean UW-QOL composite scores correlated with age (there was statistical significance between UW-QOL composite scores and age, Pearson correlation coefficient $\mathrm{r}=0.38, \mathrm{p}=$ 0.006).

Quality of Life following Total Laryngectomy

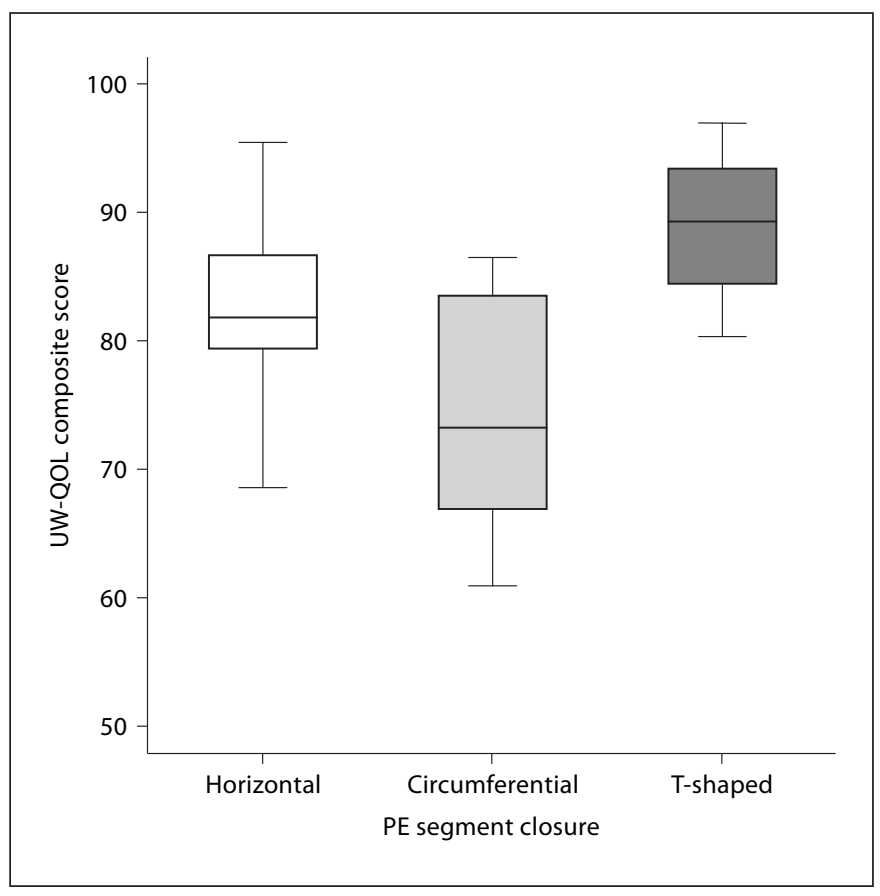

Fig. 4. Boxplot of UW-QOL composite scores correlated with the PE segment closure (data represented as median, 95\% CI and interquartile ranges; T-shaped closures were associated with significantly higher UW-QOL scores as compared to other closures, Student's t test, $\mathrm{p}<0.02$ ).

As regards overall QOL, $80 \%$ of patients cited it as very good (45.5\%) to good (34.5\%) (see table 3 and fig. 3 ). These data were essentially the same for health-related QOL.

There was no difference between the scores for men and women ( 81.8 vs. $79.1, \mathrm{p}=0.4)$. Taking 66 years as the median band, we found that the patients above the median (66-84 years) had a significantly higher mean composite score (84.5) than those below the median (78.1; Student's t test, $\mathrm{p}<0.01$; fig. 3 ). This was highlighted especially in the shoulder $(\mathrm{p}=0.01)$, recreation $(\mathrm{p}=0.004)$ and speech domains $(\mathrm{p}=0.05)$. Patients who had a Tshaped PE segment closure had a significantly higher mean composite score (89.2) as compared to those with a horizontal (81.9) or circumferential closure (74.5; Student's t test, $\mathrm{p}<0.02$; fig. 4$)$.

We found no statistical difference in the composite scores in relation to the other demographic and treatment variables such as gender, primary or recurrent cases, site of tumour, primary or secondary TOP, myotomy, nerve implantation, pharyngectomy, glossectomy, neck dissection, reconstruction, complications, radiotherapy and 
chemoradiotherapy. As many of the effects of radiotherapy and chemotherapy are similar, we re-ran the statistical tests after combining the radiation and chemotherapy groups. However, this again failed to reach statistical significance (Student's t test, $\mathrm{p}>0.05$ ). No significant difference was seen between primary surgery and secondary salvage laryngectomy groups (Student's t test, $\mathrm{p}>0.05$ ).

Amongst the domains, a number of scores demonstrated significance. Some of these findings were predictable and in keeping with our knowledge of the effects of disease and treatment in these patients. For example, scores related to saliva were significantly lower in patients who had undergone total glossectomy (Student's t test, $\mathrm{p}<0.05)$ or chemoradiation $(\mathrm{p}=0.05)$, as well as in patients with oropharyngeal tumours $(\mathrm{p}<0.05)$ or circumferential PE segment closures $(\mathrm{p}=0.01)$. Taste scores were significantly lower for total glossectomy patients $(p<0.05)$. Chewing scores were reduced in those who had had a total glossectomy $(\mathrm{p}<0.05)$. Swallowing scores were significantly lower in patients with circumferential $\mathrm{PE}$ segment closures $(\mathrm{p}=0.01)$. Shoulder scores were significantly lower in those who had had a neck dissection $(p=0.01)$. However, some other findings were less easy to explain. For example, activity scores were significantly lower in patients who had undergone circumferential PE segment closure $(\mathrm{p}=0.03)$ and in patients who had experienced complications $(\mathrm{p}=0.04)$. Chewing scores were significantly lower for those who had had a myotomy $(\mathrm{p}=0.03)$. No difference compared with $\mathrm{t}$ test data was found on verification of the results using ANOVA with Bonferroni correction.

\section{Discussion}

The impact of HNC and its treatment can have a profound effect on the patients' physical function and psychological well-being and it is essential that their perspective is taken into account. Questionnaires give a structured snapshot or insight into the patients' point of view. They facilitate multidisciplinary team working with the recognition of poor-outcome groups, better information for the patients and their carers, and the opportunity to identify problem areas and target intervention/support $[1,4]$.

Interestingly, our study group comprised patients who were experienced with valve usage (median 2 years since laryngectomy) and this may have had a bearing on the responses that we received. Experienced valve users may be better adjusted to the effects of TL on their QOL [4].
Our study had a mix of male and female patients that was representative of current incidence trends. All patients in our study were using the Blom-Singer valve in line with departmental policy. As a result, no comparisons or inferences could be drawn with other valves.

We decided to use version 4 of the UW-QOL questionnaire, an $\mathrm{HNC}$ disease-specific questionnaire, because it is well validated, short, simple to process, and proven to provide clinically relevant information, particularly in HNC [11]. However, no questionnaire is ideal for all purposes as pointed out by Ringash and Bezjak [2], and the choice for the most appropriate instrument should be individualized for each particular situation. We adopted a self-administration mode, because it avoids potential interviewer bias, is quick, simple, allows the patients to complete it at their convenience and is easy to process [4].

The results of this study demonstrate that laryngectomees have a good global long-term QOL and a high composite score. This fact suggests that the impact of TL on QOL is probably lower than one could expect and agrees with previous studies reporting that patients have good QOL and learn to cope with the disease in time $[3,5]$. These relatively high rates of patient satisfaction may reflect the perceived success of valved speech, which is superior to other techniques of alaryngeal speech $[3,4]$. This could, in part, also be a result of effective multidisciplinary team management of these patients [4]. Although the composite score (sum of domain scores) in the scale appears attractive at first, it should be viewed with caution. This is because patients tend to adjust to their disabilities which can cause a so-called 'cancellation effect' (i.e., while one score is rising, another one is falling, and the total score does not change much) [12]. We advise that the domain scores should be separately analysed and that treatment-specific effects may then become apparent through contrasting specific responses within the domains. QOL may be influenced by the time since treatment. Interestingly, however, in our series we did not find a statistically significant correlation between QOL and time since laryngectomy.

We demonstrated that speech, appearance and activity were the most important domains to patients when asked specifically. However, the influence of alaryngeal speech on QOL after TL is still controversial. Mohide et al. [15] reported that a lack of consistency exists between patients and physicians in the perception of change in QOL after treatment. In this study, physicians predicted loss of communication as the most important dimension, whereas laryngectomees reported physical consequences 
and interference with social activities as the most important issues. Palmer and Graham [10] found that the ability to communicate had the strongest association with improved QOL. In contrast, other studies have not demonstrated a correlation between QOL and speech [3]. Stewart et al. [6] assessed the relationship between voicerelated functional status and global health status and found that differences in voice handicap scores did not result in diminished QOL. Vilaseca et al. [3] identified voice handicap as a problem but stated that it was not predictive of the overall QOL.

DeSanto et al. [16] suggested that individuals who undergo TL are more concerned with the presence of the tracheostoma and interference with social activities than impaired communication. In this regard, Gritz et al. [17] noted that variables often not considered in typical QOL instruments, such as self-perceived disfigurement, selfimage, and coping style, may contribute to health functioning of individuals who are treated for HNC. Tanya and Doyle [5] reported in their series that although physical function and role were impaired after TL, this impairment was not reflected in the physical summary score.

Interestingly, although there were no statistically significant differences in the scores between men and women, the scores for the former group were higher in all the domains. These findings are in accord with data that we have generated using a new validated questionnaire designed specifically for use in laryngectomized patients [4]. These data demonstrate that females were generally less satisfied with prosthetic speech using a valve and this translated to poorer QOL values. This dissatisfaction was, at least in part, due to the low tone of prosthetic speech. Age was found to be a significant predictor of QOL outcomes with higher scores in the older age group. This could be related to the impact of vocal dysfunction on the working and social life of younger patients [4]. The reasons for the significant association with $\mathrm{PE}$ segment closure are not entirely clear.

Radiation therapy did not significantly influence the speech outcome in our sample but saliva scores were significantly reduced in those who had received chemoradiation. This finding is of great interest because of the increasing use of organ preservation protocols for advanced laryngeal cancer and the growing use of TL for failure rather than as first-line treatment [18-20]. However, here again, there have been conflicting reports in the literature. Paleri et al. [19] found no significant differences in irradiated laryngectomees and the nonirradiated group, while Vilaseca et al. [3] found that communi- cation was significantly worse when TL was the treatment for patients with treatment failure after radiation or chemoradiation.

The UW-QOL scale is a brief and simple scale that arms the clinician with useful information and can contribute to decision making based on patient feedback [11]. Moreover, it concerns issues that have been important in the last 7 days which are easy to recollect. In addition, it can help in audit, research and assist in the making of patient information leaflets/videos. The availability of open-ended text in the scale, albeit as a single item, provides yet another avenue of information regarding individual patients. We have received a variety of written comments, some of which have allowed us to recognize health care problems in need of attention that would have otherwise escaped our attention. For example, patients felt that issues such as speech, saliva, smell, cough, and mucous production were not addressed adequately in the questionnaire. This raises an important issue that existing QOL instruments may not be sensitive or specific enough for the laryngectomy population. A possible explanation for this situation could be that this is a unique group of patients with distinct problems, concerns and issues. The authors, therefore, advise the use of specific questionnaires in addition to general QOL instruments for a complete assessment. Also, the UW-QOL scale is totally subjective with no room for clinician input, has a closed format with a single open-ended question and consequently for reasons mentioned earlier does have limitations especially in its use in the laryngectomee population.

The strengths of this study include the relatively large patient number, the uniformity of surgical approach and the high response rate $(>80 \%)$. Possible weaknesses include the cross-sectional nature of the study and the risk of selection and survival bias. However, the cross-sectional technique allows a subset of surviving patients to be identified and studied utilizing a single interaction (a questionnaire). We advise prospective longitudinal studies that adequately identify changes in QOL over a period of time and, indeed, eliminate the cancellation effect to an extent. However, such prospective studies are an expensive, time- and labour-intensive enterprise. Even when thorough data collection is accomplished, other realities can compromise the usefulness of the data in the light of the unavoidable drop-outs owing to recurrence, intercurrent death, lack of motivation or loss of follow-up [12]. 


\section{Conclusion}

In summary, the composite score and overall QOL in our series of TL patients were high. Speech, activity and appearance were identified as the most important issues. Apart from age, which was a significant predictor, we found no statistical difference in the QOL scores in relation to the other demographic and treatment variables such as gender, primary or recurrent, site of tumour, pri- mary or secondary TOP, myotomy, nerve implantation, pharyngectomy, glossectomy, neck dissection, reconstruction, complications, radiotherapy and chemoradiotherapy. We strongly urge the use of prospective longitudinal studies that will adequately identify any QOL changes over time. Although the UW-QOL questionnaire is simple and brief, it has limitations that can reduce its effective use in TL patients and we advise supplementing it with the use of domain-specific questionnaires.

\section{References}

1 Jones E, Lund VJ, Howard DJ, et al: Quality of life of patients treated surgically for head and neck cancer. J Otolaryngol 1992;106: 238-242.

2 Ringash J, Bezjak A: A structured review of quality of life instruments for head and neck cancer patients. Head Neck 2001;23:201213.

-3 Vilaseca I, Chen AY, Backscheider AG: Longterm quality of life after total laryngectomy. Head Neck 2006;28:313-320.

4 Kazi R, Singh A, De-Cordova J, et al: A new self-administered questionnaire to determine patient experience with voice prosthesis (Blom-Singer valves). J Postgrad Med 2005;51:253-258

$\checkmark 5$ Tanya EL, Doyle PC: Quality of life in male tracheoesophageal speakers. J Rehabil Res Dev 2005;42:115-125.

-6 Stewart MG, Chen AY, Stach CB: Outcomes analysis of voice and quality of life in patients with laryngeal cancer. Arch Otolaryngol Head Neck Surg 1998;124:143-148.

7 Deleyiannis FW, Weymuller EA Jr, Coltrera MD, et al: Quality of life after laryngectomy: are functional disabilities important? Head Neck 1999;21:319-324.
8 Finizia C, Hammerlid E, Westin T, Lindstrom J: Quality of life and voice in patients with laryngeal carcinoma: a posttreatment comparison of laryngectomy (salvage surgery) versus radiotherapy. Laryngoscope 1998;108:1566-1573.

$\checkmark 9$ Morton RP: Studies in quality of life of head and neck cancer patients: results of a twoyear longitudinal study and a comparative cross-sectional cross-cultural survey. Laryngoscope 2003;113:1091-1103.

10 Palmer AD, Graham MS: The relationship between communication and quality of life in alaryngeal speakers. J Speech Lang Pathol Audiol 2004;28:6-24.

11 Hassan SJ, Weymuller EA: Assessment of quality of life in head and neck cancer patients. Head Neck 1993;15:485-496.

12 Weymuller EA, Yueh B, Deleyiannis FW, et al: Quality of life in patients with head and neck cancer: lessons learnt from 549 prospectively evaluated patients. Arch Otolaryngol Head Neck Surg 2000;126:329-336.

13 Laverick S, Lowe D, Brown JS, et al: The im pact of neck dissection on health-related quality of life. Arch Otolaryngol Head Neck Surg 2004;130:149-154.

14 Vartanian JG, Carvalho AL, Yueh B, et al: Long-term quality of life evaluation after head and neck cancer treatment in a developing country. Arch Otolaryngol Head Neck Surg 2004;130:1209-1213.
15 Mohide EA, Archibald SD, Tew M, et al: Postlaryngectomy quality-of-life dimensions identified by patients and health care professionals. Am J Surg 1992;164:619-622.

16 DeSanto LW, Olsen KD, Perry WC, Rohe DE, Keith RL: Quality of life after surgical treatment of cancer in the larynx. Ann Otol Rhinol Laryngol 1995;104:763-769.

17 Gritz ER, Carmack CL, de Moor C, Coscarelli A, Schacherer CW, Meyers EG, Abemayor E: First year after head and neck cancer: quality of life. J Clin Oncol 1999;17: 352-360.

18 Hanna E, Sherman A, Cash D, et al: Quality of life for patients following total laryngectomy vs chemoradiation for laryngeal preservation. Arch Otolaryngol Head Neck Surg 2004;130:875-883.

19 Paleri V, Stafford FW, Leontsinis TG, Hildreth AJ: Quality of life in laryngectomees: a post-treatment comparison of laryngectomy alone versus combined therapy. J Otolaryngol 2001;115:450-454.

20 Lotempio MM, Wang KH Sadeghi A, et al: Comparison of quality of life outcomes in laryngeal cancer patients following chemoradiation vs total laryngectomy. Otolaryngol Head Neck Surg 2005;132:948-953. 